Portland State University

PDXScholar

$10-1-1981$

\title{
Existence of the dielectric constant in dipolar fluid mixtures
}

John D. Ramshaw

Portland State University, jdramshaw@yahoo.com

Norman D. Hamer

University of New South Wales

Follow this and additional works at: https://pdxscholar.library.pdx.edu/phy_fac

Part of the Physics Commons

Let us know how access to this document benefits you.

\section{Citation Details}

J.D. Ramshaw and N.D. Hamer, "Existence of the dielectric constant in dipolar fluid mixtures," J. Chem. Phys. 75, 3511 (1981)

This Article is brought to you for free and open access. It has been accepted for inclusion in Physics Faculty Publications and Presentations by an authorized administrator of PDXScholar. Please contact us if we can make this document more accessible: pdxscholar@pdx.edu. 


\section{AIP Chemicital Prysics}

\section{Existence of the dielectric constant in dipolar fluid mixtures}

John D. Ramshaw and Norman D. Hamer

Citation: J. Chem. Phys. 75, 3511 (1981); doi: 10.1063/1.442460

View online: http://dx.doi.org/10.1063/1.442460

View Table of Contents: http://jcp.aip.org/resource/1/JCPSA6/v75/i7

Published by the American Institute of Physics.

\section{Additional information on J. Chem. Phys.}

Journal Homepage: http://jcp.aip.org/

Journal Information: http://jcp.aip.org/about/about_the_journal

Top downloads: http://jcp.aip.org/features/most_downloaded

Information for Authors: http://jcp.aip.org/authors

\section{ADVERTISEMENT}

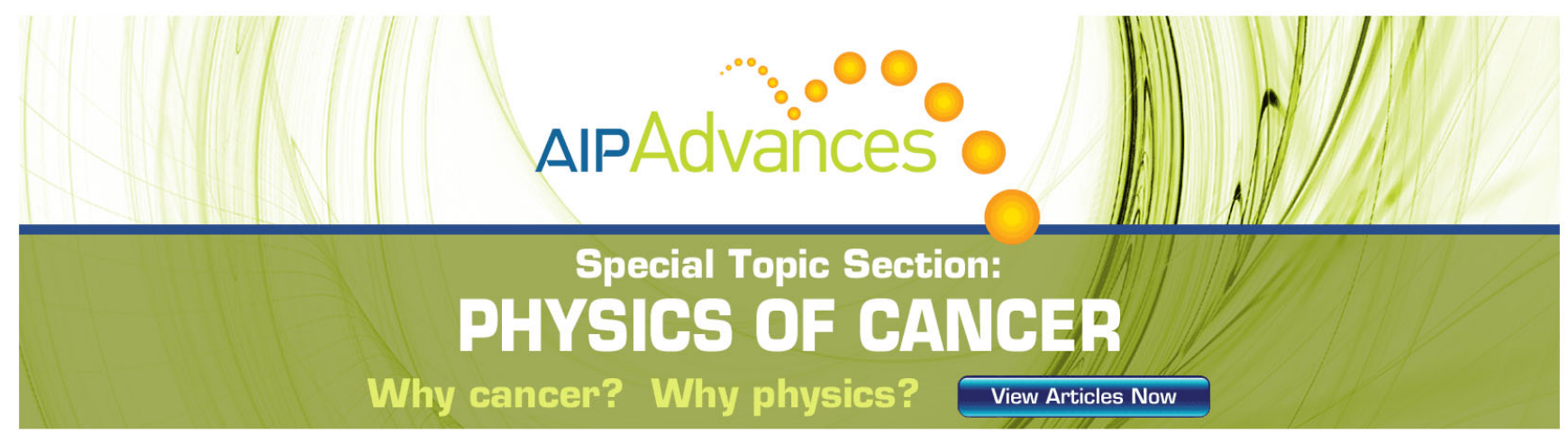




\title{
Existence of the dielectric constant in dipolar fluid mixtures $^{\text {a) }}$
}

\author{
John D. Ramshaw \\ Theoretical Division, University of California, Los Alamos National Laboratory, Los Alamos, New \\ Mexico 87545 \\ Norman D. Hamer \\ Department of Chemistry, Faculty of Military Studies, University of New South Wales, R.M.C. Duntroon, \\ A.C.T. 2600 , Australia \\ (Received 19 May 1981; accepted 16 June 1981)

\begin{abstract}
The existence of the dielectric constant $\epsilon$ is investigated for fluid mixtures of rigid polar molecules. The investigation is performed using the functional-derivative formalism for mixtures, and is closely analogous to that previously carried out for pure dipolar fluids [J. Chem. Phys. 68, 5199 (1978)]. Sufficient conditions for the existence of $\epsilon$ are obtained in terms of the direct correlation function matrix $c_{a \beta}(12)$. It is found that $\epsilon$ exists if $c_{a \beta}(12)$ depends only on relative positions and orientations, and becomes asymptotic to $-\theta_{a \beta}(12) / k T$ at long range, where $\theta_{\alpha \beta}(12)$ is the dipole-dipole potential between a molecule of species $\alpha$ and one of species $\beta$. An expression for $\epsilon$ in terms of the short-range total correlation function matrix emerges automatically from the development. This expression is equivalent to an earlier result obtained by a different method. Expressions for $\epsilon$ in terms of $c_{a \beta}(12)$ are derived for axially symmetric molecules and for molecules of arbitrary symmetry. In the former case, the expression involves the inverse of an $N_{c} \times N_{c}$ matrix, where $N_{c}$ is the number of components in the mixture. This expression facilitates the evaluation of $\epsilon$ in the mean spherical approximation. For molecules of arbitrary symmetry, the expression for $\epsilon$ in terms of $c_{a \beta}(12)$ involves the inverse of an $N_{c} \times N_{c}$ supermatrix, each element of which is a $3 \times 3$ matrix.
\end{abstract}

\section{INTRODUCTION AND SUMMARY}

The purpose of this article is to establish sufficient conditions for the existence of the dielectric constant $\epsilon$ in dipolar fluid mixtures, and to examine the form of $\epsilon$ in such mixtures in somewhat greater detail than has previously been done. Our development constitutes the extension to mixtures of previous work on pure dipolar fluids. ${ }^{1}$ As before, we restrict attention to rigid (unpolarizable) polar molecules.

The development is based on the functional-derivative formalism for fluid mixtures, and is closely analogous to that previously carried out for pure dipolar fluids. ${ }^{1}$ The functional-derivative formalism leads naturally to the introduction of the direct correlation function matrix $c_{\alpha \beta}(a b)$, in terms of which sufficient conditions for the existence of $\epsilon$ can be simply expressed. It is found that $\epsilon$ exists if $c_{\alpha \beta}(a b)$ is the sum of a short-range term that depends only on relative positions and orientations, and a long-range term- $\theta_{\alpha \beta}(a b) / k T$, where $\theta_{\alpha \beta}(a b)$ is the dipole-dipole potential between a molecule of species $\alpha$ with coordinates $(a)$ and one of species $\beta$ with coordinates (b). These conditions are straightforward mixture analogs of the sufficient conditions for the existence of $\epsilon$ in pure dipolar fluids. ${ }^{1}$

As in the single-component case, an expression for $\epsilon$ emerges automatically as a by-product of the existence theorem. The structure of the present development is such that this expression involves a short-range total correlation function matrix $h_{\alpha \beta}^{0}(a b)$, rather than $c_{\alpha \beta}(a b)$. This expression is equivalent to an earlier result ob-

\footnotetext{
a Work performed in part under the auspices of the United States Department of Energy.
}

tained, using an entirely different approach, by Martina and Deutch ${ }^{2}$ and by Hoye and Stell. ${ }^{3}$

We also derive expressions for $\epsilon$ in terms of $c_{\alpha \beta}(a b)$, first for axially symmetric molecules and then for molecules of arbitrary symmetry. In the former case, the expression involves the inverse of an $N_{c} \times N_{c}$ matrix, where $N_{c}$ is the number of components in the mixture. An equivalent expression is implicit in the work of Hoye and Stell. ${ }^{3}$ [Note that $\bar{\tau}_{i j}$ should be replaced by $\left(\tau^{-1}\right)_{i j}$ in their Eq. (16).] This expression facilitates the evaluation of $\epsilon$ in the mean spherical approximation, since it makes the explicit consideration of finite-volume effects ${ }^{4}$ unnecessary. We perform this evaluation in terms of the functions $c_{\alpha \beta}^{\Delta}(r)$ and $c_{\alpha \beta}^{ \pm}(r)$ used by Freasier, Hamer, and Isbister ${ }^{4}$ (FHI). The resulting expression for $\epsilon$ agrees with that of FHI when allowance is made for an error in their finite-volume correction.

Our expression for $\epsilon$ in terms of $c_{\alpha \beta}(a b)$ for molecules of arbitrary symmetry has not previously been presented, although $\mathrm{H}$ bye and $\mathrm{Stell}^{3}$ have indicated how this expression could be derived within the framework of their formalism. This expression involves the inverse of an $N_{c} \times N_{c}$ supermatrix, each element of which is a $3 \times 3$ matrix. The expressions for $\epsilon$ in terms of $c_{\alpha \beta}(a b)$ are more complicated than the expression involving $h_{\alpha \beta}^{0}(a b)$ because of the matrix inversions, but they have the advantage of being more easily evaluated in certain special cases, such as the mean spherical ${ }^{4}$ and linearized Debye-Hückel ${ }^{5}$ approximations.

\section{EXISTENCE OF $\epsilon$}

In this section we establish sufficient conditions for the existence of $\epsilon$ in terms of the behavior of $c_{\alpha \beta}(a b)$. 
In the process, we automatically obtain an expression for $\epsilon$ in terms of $h_{\alpha \beta}^{0}(a b)$.

We consider a finite volume $V$, of arbitrary shape, containing an arbitrary mixture of rigid (unpolarizable) polar molecules at constant temperature $T$. The number of components in the mixture is $N_{c}$, and the number of molecules of component or species $\alpha$ is $N_{\alpha}$. The number density of species $\alpha$ is $\rho_{\alpha}=N_{\alpha} / V$. The molecules of each species are of arbitrary symmetry.

The position and orientation of the $k$ th molecule of species $\alpha$ are denoted by $\mathbf{r}_{\alpha k}$ and $\omega_{\alpha k}$, respectively, and are collectively represented by the shorthand notation $(\alpha k)$. Similarly, dummy coordinates $\left(\mathbf{r}_{a}, \omega_{a}\right)$ are represented by $(a),\left(\mathrm{r}_{b}, \omega_{b}\right)$ by $(b)$, and so on. The angular measure $\int d \omega_{a}$ is denoted by $\Omega$.

To each molecule is rigidly affixed a coordinate frame which translates and rotates along with the molecule. These molecular coordinate frames are defined in the same way for all molecules of a given species, so that two such molecules coincide if their coordinate frames coincide. A molecular orientation $\omega$ is then defined by any convenient set of coordinates which specifies the orientation of the molecular frame with respect to the laboratory frame. If these coordinates are taken to be the Euler angles, ${ }^{6}$ then $\omega=(\theta, \phi, \chi)$ and $\Omega=8 \pi^{2}$. In the special case of axially symmetric molecules, the angle $\chi$ is irrelevant and we may take $\omega=(\theta, \phi)$, which makes $\Omega=4 \pi$.

Let $e_{a}^{p}=\mathrm{e}^{p}\left(\omega_{a}\right)$, where $\mathrm{e}^{p}(\omega)$ is the unit vector along the $p$ axis $(p=1,2,3$ or $x, y, z)$ of a coordinate frame with orientation $\omega$. The dipole moment vector of a molecule of species $\alpha$ with orientation $\omega_{a}$ can then be written as

$$
\mu_{\alpha_{a}}=\sum_{p} \mu_{\alpha}^{p} \mathrm{e}_{a}^{p}
$$

where $\mu_{\alpha}^{p}$ is the component of the dipole moment of a molecule of species $\alpha$ along the $p$ axis in the molecular frame. The magnitude of the molecular dipole moment for species $\alpha$ is

$$
\mu_{\alpha}=\left[\sum_{p}\left(\mu_{\alpha}^{p}\right)^{2}\right]^{1 / 2} \text {. }
$$

Our development is based upon the functional-derivative relations for fluid mixtures, which we now proceed to summarize. These relations are straightforward generalizations of those for pure fluids. ${ }^{7}$ We are concerned here only with linear dielectric polarization, so we shall require these relations only in their zero-field limiting forms. This means that we may use the grand canonical relations even though we are in the canonical ensemble, provided that the $1 / N$ correction terms in the total correlation functions are discarded. 8,9

Suppose that the sample is subjected to external fields which add a term

$$
\sum_{\alpha k} \phi_{\alpha}(\alpha k)
$$

to the total potential energy of the system. The linear (first-order) deviation $\delta n_{\alpha}(a)$ of the single-molecule generic distribution function for species $\alpha$ from its zero- field value of $\rho_{\alpha} / \Omega$ is then given by

$\delta n_{\alpha}(a)=-\beta_{T} n_{\alpha} \sum_{\beta} \int d(b) \phi_{\beta}(b)\left[n_{\beta} h_{\alpha \beta}(a b)+\delta_{\alpha \beta} \delta(a b)\right]$,

where $\beta_{T}=1 / k T, n_{\alpha}=\rho_{\alpha} / \Omega, \delta_{\alpha \beta}$ is the Kronecker delta, $\delta(a b)$ is the Dirac delta function in positions and orientations, and $h_{\alpha \beta}(a b)$ is the total correlation function matrix in zero applied field. The inverse of this relation is

$$
-\beta_{T} \phi_{\alpha}(a)=\sum_{\beta} \int d(b) \delta n_{\beta}(b)\left[-c_{\alpha \beta}(a b)+n_{\alpha}^{-1} \delta_{\alpha \beta} \delta(a b)\right],
$$

where $c_{\alpha \beta}(a b)$ is the direct correlation function matrix in zero applied field. It follows from Eqs. (3) and (4) that $h_{\alpha \beta}$ and $c_{\alpha \beta}$ are related by the Ornstein-Zernike equation for mixtures,

$$
h_{\alpha \beta}(a b)=c_{\alpha \beta}(a b)+\sum_{\gamma} n_{\gamma} \int d(e) h_{\alpha \gamma}(a e) c_{\gamma \beta}(e b) .
$$

We now specialize to the case of dielectric polarization, in which the only external field present is an external electric field $E_{0}(r)$. The latter is arbitrary except for two restrictions: it must be weak enough that the sample responds to it linearly, and it must vary slowly (in a molecular sense) with the position $r$. The external potential functions $\phi_{\alpha}(a)$ now become

$$
\phi_{\alpha}(a)=-\mu_{\alpha a} \cdot \mathrm{E}_{0}\left(\mathrm{r}_{a}\right) .
$$

The dielectric behavior of the sample is determined by the polarization (induced dipole moment per unit volume) $\mathrm{P}(\mathrm{r})$, which is related to $\delta n_{\alpha}(a)$ by

$$
\mathbf{P}\left(\mathbf{r}_{a}\right)=\sum_{\alpha} \int d \omega_{a} \delta n_{\alpha}(a) \mu_{\alpha a}
$$

Sufficient conditions for the existence of $\epsilon$ are most easily expressed in terms of the direct correlation function matrix $c_{\alpha \beta}(a b)$. We adopt two fundamental assumptions about its behavior:

(i) We assume that at long range (large $\left.\left|\mathbf{r}_{a}-\mathbf{r}_{b}\right|\right)$, $c_{\alpha \beta}(a b)$ becomes asymptotic to $-\beta_{T}$ times the dipoledipole potential between a molecule of species $\alpha$ with coordinates $(a)$ and one of species $\beta$ with coordinates (b). That is,

$$
\begin{aligned}
& c_{\alpha \beta}(a b)=c_{\alpha \beta}^{0}(a b)-\beta_{T} \theta_{\alpha \beta}(a b), \\
& \theta_{\alpha \beta}(a b)=-\mu_{\alpha a} \cdot \mathbf{T}_{0}\left(\mathbf{r}_{a}-\mathbf{r}_{b}\right) \cdot \mu_{\beta b}, \\
& \mathbf{T}_{0}(\mathbf{r})=H(|\mathbf{r}|-\sigma) \nabla \nabla|\mathbf{r}|^{-1},
\end{aligned}
$$

where $c_{\alpha \beta}^{0}(a b)$ is a short-ranged function, $H(x)$ is the Heaviside unit step function, and it is understood that the limit $\sigma \rightarrow 0$ is ultimately to be taken.

(ii) We assume that $c_{\alpha \beta}^{0}(a b)$ depends only on relative positions and orientations.

These assumptions are entirely analogous to those which imply the existence of $\epsilon$ in pure dipolar fluids. ${ }^{1}$ In an infinite system, the validity of (i) may be inferred from the work of Hoye and Stell, ${ }^{3}$ and that of (ii) is a trivial consequence of translational and rotational invariance. In the finite systems of present concern, the validity of (i) and (ii) is very plausible (except of course in a negligibly thin surface layer, where (ii) will break down) but as yet unproved, and we shall not pursue the 
question here. We shall simply show that (i) and (ii) do indeed imply the existence of $\epsilon$ in dipolar fluid mixtures.

Combining Eqs. (4), (6), and (8), we obtain

$\beta_{T} \mu_{\alpha a} \cdot \mathrm{E}_{L}\left(\mathrm{r}_{a}\right)=\sum_{\beta} \int d(b) \delta n_{\beta}(b)\left[-c_{\alpha \beta}^{0}(a b)+n_{\alpha}^{-1} \delta_{\alpha \beta} \delta(a b)\right]$,

where

$$
\mathbf{E}_{L}(\mathbf{r})=\mathbf{E}_{0}(\mathbf{r})+\int d \mathbf{r}^{\prime} \mathbf{T}_{0}\left(\mathbf{r}-\mathbf{r}^{\prime}\right) \cdot \mathbf{P}\left(\mathbf{r}^{\prime}\right)
$$

is the Lorentz electric field, which is related to the Maxwell electric field $E(r)$ by $E_{L}(r)=E(r)+(4 \pi / 3) P(r)$. In order to invert Eq. (11), we introduce a short-ranged total correlation function matrix $h_{\alpha \beta}^{0}(a b)$ defined by

$$
h_{\alpha \beta}^{0}(a b)=c_{\alpha \beta}^{0}(a b)+\sum_{\gamma} n_{\gamma} \int d(e) h_{\alpha \gamma}^{0}(a e) c_{\gamma \beta}^{0}(e b) .
$$

This definition enables Eq. (11) to be solved for $\delta n_{\alpha}(a)$, with the result

$$
\delta n_{\alpha}(a)=\beta_{T} n_{\alpha} \sum_{\beta} \int d(b)\left[n_{\beta} h_{\alpha \beta}^{0}(a b)+\delta_{\alpha \beta} \delta(a b)\right] \mu_{\beta b} \cdot \mathrm{E}_{L}\left(\mathrm{r}_{b}\right) .
$$

Since $c_{\alpha \beta}^{0}(a b)$ is short ranged, so is $h_{\alpha \beta}^{0}(a b)$. The spatial integrals over $r_{b}$ in Eqs. (11) and (14) may therefore be extended over all space instead of just over the volume $V$, provided that $r_{a}$ is farther than the range of $c_{\alpha \beta}^{0}(a b)$ or $h_{\alpha \beta}^{0}(a b)$ from the surface of $V$. The integral over $r_{e}$ in Eq. (13) may similarly be extended over all space. In contrast, Eqs. (3), (4), and (5) involve the longranged functions $c_{\alpha \beta}(a b)$ and $h_{\alpha \beta}(a b)$, and the spatial integrals in these equations extend only over the sample volume $V$.

Since $E_{0}(r)$ is slowly varying and the system is a fluid, it is clear that $P(r)$ and hence $E_{L}(r)$ will also be slowly varying. Combined with the fact that $h_{\alpha \beta}^{0}(a b)$ is short ranged, this means that $E_{L}\left(r_{b}\right)$ in Eq. (14) may be evaluated at the point $r_{b}=r_{a}$ and taken outside the integral over $(b)$. When this is done, the result combines with Eq. (7) to yield

$$
\mathbf{P}\left(\mathbf{r}_{a}\right)=\mathbf{A}\left(\mathbf{r}_{a}\right) \cdot \mathbf{E}_{L}\left(\mathbf{r}_{a}\right),
$$

where

$$
\mathbf{A}\left(\mathbf{r}_{a}\right)=\beta_{T} \sum_{\alpha \beta} n_{\alpha} \int d \mathbf{r}_{b} d \omega_{a} d \omega_{b}\left[n_{\beta} h_{\alpha \beta}^{0}(a b)+\delta_{\alpha \beta} \delta(a b)\right] \mu_{\alpha a} \mu_{B b} .
$$

But since $c_{\alpha \beta}^{0}(a b)$ depends only on relative positions and orientations according to (ii), it is clear from Eq. (13) that the same is true for $h_{\alpha \beta}^{0}(a b)$. By symmetry, there fore, $A\left(r_{a}\right)$ must be independent of $r_{a}$ and proportional to the unit tensor $\mathbf{U}$. Thus $\mathbf{A}\left(\mathbf{r}_{a}\right)=A \mathbf{U}$, and $A=(1 / 3) \mathbf{A}: \mathbf{U}$. Equation (15) now reduces to $\mathrm{P}(\mathrm{r})=A \mathrm{E}_{L}(\mathrm{r})$, which shows ${ }^{10}$ that the dielectric constant exists and is given by $(\epsilon-1) /$ $(\epsilon+2)=4 \pi A / 3$; i. e. ,

$$
\begin{aligned}
\frac{\epsilon-1}{\epsilon+2}= & \frac{4 \pi}{9} \beta_{T} \sum_{\alpha \beta} n_{\alpha} \int d \mathbf{r}_{b} d \omega_{a} d \omega_{b} \\
& \times\left[n_{\beta} h_{\alpha \beta}^{0}(a b)+\delta_{\alpha \beta} \delta(a b)\right] \mu_{\alpha a} \cdot \mu_{B b} .
\end{aligned}
$$

We have therefore shown that assumptions (i) and (ii) imply the existence of $\epsilon$ in dipolar fluid mixtures, with $\epsilon$ given by Eq. (17). This expression for $\epsilon$ is equivalent to that obtained by Martina and Deutch ${ }^{2}$ and Hoye and Stell $^{3}$ using an entirely different method. [These authors' $W_{\alpha \beta}(a b)$ is the same as our $\rho_{\alpha} \rho_{\beta} h_{\alpha \beta}^{0}(a b)$. ]

\section{III. $\epsilon$ IN TERMS OF $c_{\alpha \beta}(a b)$ FOR MOLECULES OF AXIAL SYMMETRY}

In this section we derive the expression for $\epsilon$ in terms of $c_{\alpha \beta}(a b)$ for axially symmetric molecules. To this end, we define

$$
\begin{aligned}
& m_{\alpha}=n_{\alpha}^{1 / 2} \mu_{\alpha}, \\
& H_{\alpha \beta}=\frac{\left(n_{\alpha} n_{\beta}\right)^{1 / 2}}{\Omega \mu_{\alpha} \mu_{\beta}} \int d \mathrm{r}_{b} d \omega_{a} d \omega_{b} h_{\alpha \beta}^{0}(a b) \mu_{\alpha a} \cdot \mu_{B b} .
\end{aligned}
$$

Equation (17) can now be rewritten as

$$
\begin{aligned}
\frac{\epsilon-1}{\epsilon+2} & =\frac{4 \pi}{9} \beta_{T} \Omega \sum_{\alpha \beta} m_{\alpha}\left(\delta_{\alpha \beta}+H_{\alpha \beta}\right) m_{\beta} \\
& =\frac{4 \pi}{9} \beta_{T} \Omega\left[\hat{m}^{T}(\hat{I}+\hat{H}) \hat{m}\right],
\end{aligned}
$$

where $\hat{m}$ is the $N_{c}$-dimensional column vector with elements $m_{\alpha}, \hat{m}^{T}$ is the corresponding row vector, $\hat{I}$ is the $N_{c} \times N_{c}$ unit matrix with elements $\delta_{\alpha \beta}$, and $\hat{H}$ is the $N_{c}$ $\times N_{c}$ matrix with elements $H_{\alpha \beta}$.

Multiply Eq. (13) by $\left(\Omega \mu_{\alpha} \mu_{\beta}\right)^{-1}\left(n_{\alpha} n_{\beta}\right)^{1 / 2} \mu_{\alpha a} \cdot \mu_{B b}$ and integrate over $r_{b}, \omega_{a}$, and $\omega_{b}$. The result is

$$
\begin{aligned}
H_{\alpha \beta}= & C_{\alpha \beta}+\frac{\left(n_{\alpha} n_{\beta}\right)^{1 / 2}}{\Omega \mu_{\alpha} \mu_{\beta}} \\
& \times \sum_{\gamma} n_{\gamma} \int d \omega_{a} d(b) d(e) h_{\alpha \gamma}^{0}(a e) c_{\gamma \beta}^{0}(e b) \mu_{\alpha a} \cdot \mu_{\beta b},
\end{aligned}
$$

where

$$
c_{\alpha \beta}=\frac{\left(n_{\alpha} n_{\beta}\right)^{1 / 2}}{\Omega \mu_{\alpha} \mu_{\beta}} \int d \mathbf{r}_{b} d \omega_{a} d \omega_{b} c_{\alpha \beta}^{0}(a b) \mu_{\alpha a} \cdot \mu_{\beta b} .
$$

We note that $c_{\alpha \beta}^{0}(a b)$ may be replaced by $c_{\alpha \beta}(a b)$ in Eq.

(22) without changing $C_{\alpha \beta}$, so we can equally well write

$$
C_{\alpha \beta}=\frac{\left(n_{\alpha} n_{\beta}\right)^{1 / 2}}{\Omega \mu_{\alpha} \mu_{\beta}} \int d \mathbf{r}_{b} d \omega_{a} d \omega_{b} c_{\alpha \beta}(a b) \mu_{\alpha a} \cdot \mu_{\beta b} .
$$

The proof that this replacement is legitimate will be omitted, as it is entirely analogous to that of the corresponding property for pure dipolar fluids. ${ }^{1}$

The axial molecular symmetry, together with (ii), implies that $\int d(b) c_{\gamma \beta}^{0}(e b) \mu_{\beta b}=D_{\gamma \beta} \mu_{\gamma e}$, where $D_{\gamma \beta}$ is a constant independent of $(e)$. Its value may be determined by taking the dot product with $\mu_{\gamma e}$, and integrating over $\omega_{B}$ for good measure. One thereby finds $D_{\gamma \beta}=\left(n_{\gamma} n_{\beta}\right)^{-1 / 2}$ $\times\left(\mu_{\beta} / \mu_{\gamma}\right) C_{\gamma \beta}$, so that

$$
\int d(b) c_{\gamma \beta}^{0}(e b) \mu_{B b}=\left(n_{\gamma} n_{B}\right)^{-1 / 2}\left(\mu_{B} / \mu_{\gamma}\right) c_{\gamma B} \mu_{\gamma e} .
$$

With this result, Eq. (21) reduces to

$$
H_{\alpha \beta}=C_{\alpha \beta}+\sum_{\gamma} H_{\alpha \gamma} C_{\gamma \beta},
$$

or in matrix notation

$$
\hat{H}=\hat{C}+\hat{H} \hat{C},
$$

where $\hat{C}$ is the $N_{c} \times N_{c}$ matrix with elements $C_{\alpha \beta}$. It fol- 
lows from Eq. (26) that $(\hat{I}+\hat{H})=(\bar{I}-\hat{C})^{-1}$, which combines with Eq. (20) to yield

$$
\begin{aligned}
\frac{\epsilon-1}{\epsilon+2} & =\frac{4 \pi}{9} \beta_{T} \Omega\left[\hat{m}^{T}(\hat{I}-\hat{C})^{-1} \hat{m}\right] \\
& =\frac{4 \pi}{9} \beta_{T} \Omega \sum_{\alpha \beta} m_{\alpha}(\hat{I}-\hat{C})_{\alpha \beta}^{-1} m_{\beta} .
\end{aligned}
$$

Equation (27) is the expression for $\epsilon$ in terms of $c_{\alpha \beta}(a b)$ for fluid mixtures of dipolar molecules with axial symmetry. An equivalent expression is implicit in the work of Hoye and Stell. ${ }^{3}$

\section{IV. $\epsilon$ IN TERMS OF $c_{\alpha \beta}(a b)$ FOR MOLECULES OF ARBITRARY SYMMETRY}

The development here follows the same outline as that of the preceding section, but is complicated by the fact that it is now necessary to resolve the dipole moment vectors into their components using Eq. (1). Let

$$
\begin{aligned}
& m_{\alpha}^{p}=n_{\alpha}^{1 / 2} \mu_{\alpha}^{p}, \\
& H_{\alpha \beta}^{p \alpha}=\Omega^{-1}\left(n_{\alpha} n_{\beta}\right)^{1 / 2} \int d \mathrm{r}_{b} d \omega_{a} d \omega_{b} h_{\alpha \beta}^{0}(a b) \mathrm{e}_{a}^{p} \cdot \mathrm{e}_{b}^{q},
\end{aligned}
$$

so that Eq. (17) can be rewritten in the form

$$
\begin{aligned}
\frac{\epsilon-1}{\epsilon+2} & =\frac{4 \pi}{9} \beta_{T} \Omega \sum_{\alpha \beta p q} m_{\alpha}^{p}\left(\delta_{\alpha \beta} \delta_{p q}+H_{\alpha \beta}^{p q}\right) m_{\beta}^{q} \\
& =\frac{4 \pi}{9} \beta_{T} \Omega\left[\tilde{m}^{T}(\tilde{I}+\tilde{H}) \tilde{m}\right] .
\end{aligned}
$$

Here $\bar{m}$ is an $N_{c}$-dimensional column supervector, whose $\alpha$ th component is a three-dimensional column vector with components $m_{\alpha}^{p} ; \tilde{m}^{T}$ is the corresponding row supervector; $\tilde{H}$ is an $N_{c} \times N_{c}$ supermatrix whose $\alpha \beta$ element is a $3 \times 3$ matrix with elements $H_{\alpha \beta}^{p q}$; and $\tilde{I}$ is the unit $N_{c} \times N_{c}$ supermatrix, whose $\alpha \beta$ element is the $3 \times 3$ matrix with elements $\delta_{\alpha \beta} \delta_{p q}$. One readily verifies that the multiplication rules for such supervectors and supermatrices are precisely those embodied in Eq. (30).

Now multiply Eq. (13) by $\Omega^{-1}\left(n_{\alpha} n_{\beta}\right)^{1 / 2} e_{a}^{p} \cdot e_{b}^{q}$ and integrate over $r_{b}, \omega_{a}$, and $\omega_{b}$ to obtain

$$
\begin{aligned}
H_{\alpha \beta}^{p a}= & C_{\alpha \beta}^{p \alpha}+\Omega^{-1}\left(n_{\alpha} n_{\beta}\right)^{1 / 2} \\
& \times \sum_{\gamma} n_{\gamma} \int d \omega_{a} d(b) d(e) h_{\alpha \gamma}^{0}(a e) c_{\gamma \beta}^{0}(e b) \mathrm{e}_{a}^{p} \cdot \mathrm{e}_{b}^{q},
\end{aligned}
$$

where

$$
\begin{aligned}
C_{\alpha \beta}^{p a} & =\Omega^{-1}\left(n_{\alpha} n_{\beta}\right)^{1 / 2} \int d \mathrm{r}_{b} d \omega_{a} d \omega_{b} c_{\alpha \beta}^{0}(a b) \mathrm{e}_{a}^{p} \cdot \mathbf{e}_{b}^{q} \\
& =\Omega^{-1}\left(n_{\alpha} n_{\beta}\right)^{1 / 2} \int d \mathrm{r}_{b} d \omega_{a} d \omega_{b} c_{\alpha \beta}(a b) \mathrm{e}_{a}^{p} \cdot \mathbf{e}_{b}^{q} .
\end{aligned}
$$

The second equality in Eq. (32) expresses the fact that $c_{\alpha \beta}^{0}(a b)$ may be replaced by $c_{\alpha \beta}(a b)$ without changing $C_{\alpha \beta}^{\phi \alpha}$; the proof is again analogous to that for pure dipolar fluids, ${ }^{1}$ and hence is omitted. It should perhaps be noted that the analogous replacement of $h_{\alpha \beta}^{0}(a b)$ by $h_{\alpha \beta}(a b)$ in Eqs. (19) and (29) is not permissible.

Because of (ii), the quantity $\int d(b) c_{\gamma \beta}^{0}(e b) \mathbf{e}_{b}^{\alpha}$ must be a fixed vector in the molecular frame with orientation $\omega_{\theta}$; it is therefore of the form $\sum_{r} D_{\gamma B}^{r q} \mathrm{e}_{e}^{r}$, where $D_{\gamma B}^{r a}$ are constants independent of $(e)$. These constants may be deter- mined by taking the dot product with $e_{e}^{s}$, and integrating over $\omega_{\beta}$ for good measure. This yields $D_{\gamma \beta}^{r q}=\left(n_{\gamma} n_{\beta}\right)^{-1 / 2}$ $\times C_{\gamma \beta}^{r q}$, so that

$$
\int d(b) c_{\gamma B}^{0}(e b) \mathrm{e}_{b}^{q}=\left(n_{\gamma} n_{B}\right)^{-1 / 2} \sum_{\gamma} c_{\gamma B}^{r a} \mathrm{e}_{e}^{r} .
$$

This result now combines with $\mathrm{E}_{\mathbf{q}}$. (31) to give

$$
H_{\alpha \beta}^{p a}=C_{\alpha \beta}^{p a}+\sum_{\gamma \gamma} H_{\alpha \gamma}^{p r} C_{\gamma \beta}^{r q}
$$

or in supermatrix notation

$$
\tilde{H}=\tilde{C}+\tilde{H} \tilde{C},
$$

where $\tilde{C}$ is the $N_{c} \times N_{c}$ supermatrix whose $\alpha \beta$ element is the $3 \times 3$ matrix with elements $C_{\alpha \beta}^{p \alpha}$. It follows from Eq. (35) that $(\tilde{I}+\tilde{H})=(\tilde{I}-\tilde{C})^{-1}$, whence Eq. (30) becomes

$$
\begin{aligned}
\frac{\epsilon-1}{\epsilon+2} & =\frac{4 \pi}{9} \beta_{T} \Omega\left[\tilde{m}^{T}(\tilde{I}-\tilde{C})^{-1} \tilde{m}\right] \\
& =\frac{4 \pi}{9} \beta_{T} \Omega \sum_{\alpha \beta p q} m_{\alpha}^{p}\left[(\tilde{I}-\tilde{C})^{-1}\right]_{\alpha \beta}^{p \alpha} m_{\beta}^{q} .
\end{aligned}
$$

Equation (36) is the expression for $\epsilon$ in terms of $c_{\alpha \beta}(a b)$ for dipolar fluid mixtures with arbitrary molecular symmetry. This expression does not appear to have been previously presented, although Hoye and Stell $^{3}$ have indicated how an equivalent expression could be derived in their theory.

\section{THE MEAN SPHERICAL APPROXIMATION}

In this section we show how the expression for $\epsilon$ in terms of $c_{\alpha \beta}(a b)$ may be used to evaluate $\epsilon$ in the mean spherical approximation (MSA) in terms of known functions. Since the MSA entails axial molecular symmetry, the appropriate equations are those of Sec. III.

The evaluation of $\epsilon$ for dipolar mixtures in the MSA has recently been considered by Freasier, Hamer, and Isbister ${ }^{4}$ (FHI). These authors performed the evaluation by generalizing Wertheim's procedure ${ }^{11}$ to the case of mixtures. Unfortunately, this procedure involves a rather cumbersome finite-volume correction, which compensates for the fact that the system is finite rather than infinite. In the present development, however, no such correction is necessary; finite-volume effects are automatically accounted for by the fact that the spatial integral in the Ornstein-Zernike equation (5) extends only over the sample volume $V$. We may therefore evaluate $\epsilon$ simply by substituting the MSA expression for $c_{\alpha \beta}(a b)$ into Eq. (23) and combining the result with Eq. (27).

The MSA solution for $c_{\alpha \beta}(a b)$ is of the form ${ }^{4}$

$$
\begin{aligned}
c_{\alpha \beta}(a b)= & c_{\alpha \beta}^{s}\left(\left|\mathbf{r}_{a b}\right|\right)+\left(\mu_{\alpha} \mu_{\beta}\right)^{-1} c_{\alpha \beta}^{\Delta}\left(\left|\mathbf{r}_{a b}\right|\right) \mu_{\alpha a} \cdot \mu_{\beta b} \\
& +\left(\mu_{\alpha} \mu_{\beta}\right)^{-1} c_{\alpha \beta}^{D}\left(\left|\mathbf{r}_{a b}\right|\right) \mu_{\alpha a} \mu_{B b}:\left(3\left|\mathbf{r}_{a b}\right|^{-2} \mathbf{r}_{a b} \mathbf{r}_{a b}-\mathbf{U}\right),
\end{aligned}
$$

where $\mathbf{r}_{a b}=\mathbf{r}_{a}-\mathbf{r}_{b}$. Equation (37) combines with Eq. (23) to yield

$$
C_{\alpha \beta}=(4 \pi / 3)\left(\rho_{\alpha} \rho_{\beta}\right)^{1 / 2} \int_{0}^{\infty} r^{2} d r c_{\alpha \beta}^{\Delta}(r),
$$

where use has been made of the easily verified relation 
$\int d \omega_{a} \mu_{\alpha a} \mu_{\alpha_{a}}=(\Omega / 3) \mu_{\alpha}^{2} U$. In terms of the conventional functions ${ }^{4} c_{\alpha \beta}^{ \pm}(r)$, Eq. (38) becomes

$$
C_{\alpha \beta}=(8 \pi / 3)\left(\rho_{\alpha} \rho_{\beta}\right)^{1 / 2} \int_{0}^{\infty} r^{2} d r\left[c_{\alpha \beta}^{+}(r)-c_{\alpha \beta}^{-}(r)\right]
$$

The MSA result for $\epsilon$ is now determined by Eq. (27), with $C_{\alpha \beta}$ given by either Eq. (38) or Eq. (39).

We must now compare our MSA result for $\epsilon$ with that found by FHI. The FHI result is obtained by combining their Eqs. (2.60) and (2.62). Their quantities $\tilde{c}_{\alpha \theta_{,} \infty}^{ \pm}(k=0)$ correspond to our quantities $4 \pi \int r^{2} d r c_{\alpha \beta}^{ \pm}(r)$. We note, however, that their Eq. (2.60) for the finite-volume correction is incorrect due to an error in transcription; the correct equation is obtained by replacing $y_{\alpha \beta}^{ \pm}$with $-y_{\alpha \beta}^{\prime}$. When this is done, it becomes a simple matter to verify that their result is precisely equivalent to our Eqs. (27) and (39). (Note that the result in this form is not restricted to binary mixtures.)

\section{ACKNOWLEDGMENT}

One of us (N.D.H.) would like to thank Professor S. H. Lin of Arizona State University for hospitality extended while this work was in progress.

${ }^{1}$ J. D. Ramshaw, J. Chem. Phys. 68, 5199 (1978), and references cited therein.

${ }^{2}$ E. Martina and J. M. Deutch, Chem. Phys. 27, 183 (1978).

${ }^{3}$ J. S. Hóye and G. Stell, J. Chem. Phys. 70, 2894 (1979).

${ }^{4}$ B. Freasier, N. Hamer, and D. Isbister, Mol. Phys. 38, 1661 (1979).

${ }^{5}$ J. D. Ramshaw, J. Chem. Phys. 73, 3695 (1980).

${ }^{6} \mathrm{H}$. Goldstein, Classical Mechanics, 2nd ed. (Addison-Wesley, Reading, Mass., 1980).

${ }^{7}$ G. S. Rushbrooke, in Physics of Simple Liquids, edited by H. N. V. Temperley, J. S. Rowlinson, and G. S. Rushbrooke (Wiley-Interscience, New York, 1968), p. 25.

${ }^{8}$ J. D. Ramshaw, Mol. Phys. 41, 219 (1980).

${ }^{9}$ J. D. Ramshaw, J. Chem. Phys. 73, 5294 (1980).

${ }^{10}$ J. D. Ramshaw, J. Chem. Phys. 57, 2684 (1972).

${ }^{11}$ M. S. Wertheim, J. Chem. Phys. 55, 4291 (1971). 